Made available courtesy of EDP Sciences: http://publications.edpsciences.org/

***Reprinted with permission. No further reproduction is authorized without written permission from EDP Sciences.

This version of the document is not the version of record. Figures and/or pictures may be missing from this format of the document.***

\title{
A NOTE ON THE NUMBER OF SQUARES IN A PARTIAL WORD WITH ONE HOLE*
}

\author{
Francine Blanchet-SADri ${ }^{1}$ AND RoBERT MERCAŞ ${ }^{2}$
}

\begin{abstract}
A well known result of Fraenkel and Simpson states that the number of distinct squares in a word of length $n$ is bounded by $2 n$ since at each position there are at most two distinct squares whose last occurrence starts. In this paper, we investigate squares in partial words with one hole, or sequences over a finite alphabet that have a "do not know" symbol or "hole". A square in a partial word over a given alphabet has the form $u v$ where $u$ is compatible with $v$, and consequently, such square is compatible with a number of words over the alphabet that are squares. Recently, it was shown that for partial words with one hole, there may be more than two squares that have their last occurrence starting at the same position. Here, we prove that if such is the case, then the length of the shortest square is at most half the length of the third shortest square. As a result, we show that the number of distinct squares compatible with factors of a partial word with one hole of length $n$ is bounded by $\frac{7 n}{2}$.
\end{abstract}

Mathematics Subject Classification. 68R15, 05A05.

\section{INTRODUCTION}

A well known problem is the determination of the maximum number of distinct squares in any word of length $n$. With this problem progress has been made: Fraenkel and Simpson showed that this number is at most $2 n$ [6], a result recently proved somewhat more simply by Ilie [7], then later improved to

\footnotetext{
Keywords and phrases. Combinatorics on words, partial words, squares.

* This material is based upon work supported by the National Science Foundation under Grant No. DMS-0754154. The Department of Defense is also gratefully acknowledged. We thank Abraham Rashin and Kristen Wetzler for useful suggestions. An Internet server interface at www. uncg. edu/cmp/research/squares has been established for automated use of the program.

${ }^{1}$ Department of Computer Science, University of North Carolina, P.O. Box 26170, Greensboro, NC 27402-6170, USA; blanchet@uncg.edu

2 GRLMC, Universitat Rovira i Virgili, Departament de Filologies Romàniques,

Av. Catalunya 35, Tarragona, 43002, Spain
} 
$2 n-\Theta(\log n)$ [8]. Experiment strongly suggests that this number is less than $n$. In order to show this, one needs to somehow limit to less than one the average number of squares whose last occurrence begins at the positions of the word.

In this paper, we investigate the problem of counting distinct squares in partial words with one hole, or sequences over a finite alphabet that may contain a "do not know" symbol or "hole". The counting of distinct squares in partial words was recently initiated by Blanchet-Sadri et al. and revealed surprising results [4]. There, it was shown that for partial words with one hole, there may be more than two squares that have their last occurrence starting at the same position, and that if such is the case, then the hole is in the shortest square. As discussed in [4], although computations show that the actual bound for one-hole partial words of length $n$ is at most $n$ distinct squares, the results obtained there using the approach of Fraenkel and Simpson make the bound directly dependable on the size of the alphabet. Finding a dependency between the maximum number of squares starting at one position and the length of the word might be a solution. Solving this problem, at least partially, could also give a new perspective to the study of the maximum number of distinct squares in words without holes.

In Section 2, we will prove that if three squares have their last occurrence starting at the same position in a partial word with one hole, then the length of the shortest square is at most half the length of the third shortest square. As a result, we will show that the number of distinct squares compatible with factors of a partial word with one hole of length $n$ is bounded by $\frac{7 n}{2}$. In Section 3 , we will conclude with some remarks on the positions where three or more squares have their last occurrences.

In the rest of this section, we review basic concepts on partial words. Fixing a nonempty finite set of letters or an alphabet $A$, a partial word $u$ of length $|u|=n$ over $A$ is a partial function $u:\{0, \ldots, n-1\} \rightarrow A$. For $0 \leq i<n$, if $u(i)$ is defined, then $i$ belongs to the domain of $u$, denoted by $i \in D(u)$, otherwise $i$ belongs to the set of holes of $u$, denoted by $i \in H(u)$. The unique word of length 0 , denoted by $\varepsilon$, is called the empty word. For convenience, we will refer to a partial word over $A$ as a word over the enlarged alphabet $A_{\diamond}=A \cup\{\diamond\}$, where $\diamond \notin A$ represents a hole. The set of all words (respectively, partial words) over $A$ of finite length is denoted by $A^{*}$ (respectively, $A_{\diamond}^{*}$ ).

The partial word $u$ is contained in the partial word $v$, denoted by $u \subset v$, provided that $|u|=|v|$, all elements in $D(u)$ are in $D(v)$, and for all $i \in D(u)$ we have that $u(i)=v(i)$. The partial words $u$ and $v$ are compatible, denoted by $u \uparrow v$, provided that there exists a partial word $w$ such that $u \subset w$ and $v \subset w$. An equivalent formulation of compatibility is that $|u|=|v|$ and for all $i \in D(u) \cap D(v)$ we have that $u(i)=v(i)$. The following rules are useful for computing with partial words: (1) Multiplication: if $u \uparrow v$ and $x \uparrow y$, then $u x \uparrow v y$; (2) Simplification: if $u x \uparrow v y$ and $|u|=|v|$, then $u \uparrow v$ and $x \uparrow y$; and (3) Weakening: if $u \uparrow v$ and $w \subset u$, then $w \uparrow v$. If $u, v$ are nonempty compatible partial words, then $u v$ is called a square. Whenever we refer to a square $u v$ it will imply that $u \uparrow v$. 
A period of a partial word $u$ is a positive integer $p$ such that $u(i)=u(j)$ whenever $i, j \in D(u)$ and $i \equiv j \bmod p$. In this case, we call $u$ p-periodic. A weak period of $u$ is a positive integer $p$ such that $u(i)=u(i+p)$ whenever $i, i+p \in D(u)$. In this case, we call $u$ weakly p-periodic. Note that every weakly $p$-periodic word is $p$-periodic but this is not necessarily true for partial words.

For partial words $u, v, w$, if $w=u v$, then $u$ is a prefix of $w$, denoted by $u \leq w$, and if $v \neq \varepsilon$, then $u$ is a proper prefix of $w$, denoted by $u<w$. If $w=x u y$, then $u$ is a factor of $w$.

\section{BOUND ON THE NUMBER OF SQUARES}

At each position in a word there are at most two distinct squares whose last occurrence starts, and thus the following theorem holds (a short proof is given in [7]).

Theorem 2.1 [6]. Any word of length $n$ has at most $2 n$ distinct squares.

We now consider the one-hole case which behaves very differently from the zerohole case. We will also count each square at the position where its last occurrence starts. If the last occurrence of a square in a partial word starts at position $i$, then it is a square at position $i$. In the case of partial words with one hole, there may be more than two squares that have their last occurrence starting at the same position. Such is the case with $a \diamond a a b a b a a b$ that has three squares at position 0 : $(a a)^{2},(a b a)^{2}$ and $(a b a a b)^{2}$. Now, if we consider the word $a \diamond a a b a b a a b a a a a$, the square $(a a)^{2}$ has occurrences starting at positions 0 and 10 . So $(a a)^{2}$ is not a square at position 0 , but it is a square at position 10 since its last occurrence starts at position 10 .

Theorem 2.2 [4]. If a partial word with one hole has at least three distinct squares at the same position, then the hole is in the shortest square.

The following lemma extends Fine and Wilf's periodicity result to partial words with one hole.

Lemma 2.1 [1]. Let $w \in A_{\diamond}^{*}$ be weakly p-periodic and weakly q-periodic. If $H(w)$ is a singleton and $|w| \geq p+q$, then $w$ is (strongly) $\operatorname{gcd}(p, q)$-periodic.

The following lemmas on commutativity and conjugacy will also be useful for our purposes.

Lemma 2.2 [1]. Let $x, y \in A^{+}$and let $z \in A_{\diamond}^{*}$ be such that $H(z)$ is a singleton. If $z \subset x y$ and $z \subset y x$, then $x y=y x$.

Lemma 2.3 [5]. Let $x, y, z \in A_{\diamond}^{*}$ be such that $|x|=|y|>0$. Then $x z \uparrow z y$ if and only if $x z y$ is weakly $|x|$-periodic.

Lemma 2.4 [3]. Let $x, y \in A_{\diamond}^{+}$and $z \in A^{*}$. If $x z \uparrow z y$, then there exist $v, w \in A^{*}$ and an integer $n \geq 0$ such that $x \subset v w, y \subset w v$, and $z=(v w)^{n} v$. Consequently, if $x z \uparrow z y$, then $x z y$ is (strongly) $|x|$-periodic. 


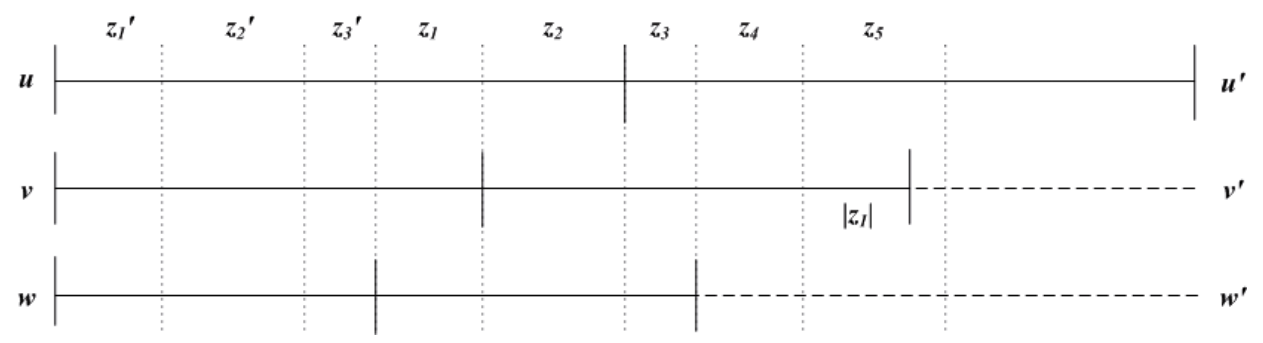

Figure 1. The case when $\left|w w^{\prime}\right|>|u|$.

Theorem 2.3. Let $w w^{\prime}, v v^{\prime}$ and $u u^{\prime}$ be three squares at the same position, with $|w|<|v|<|u|$. If $H\left(u u^{\prime}\right)$ is a singleton, then $\left|w w^{\prime}\right| \leq|u|$.

Proof. Since $|w|<|v|<|u|$, let us denote $v=w z_{1}$ and $u=v z_{2}$, for some partial words $z_{1}, z_{2}$ over the alphabet $A$. By contradiction, let us assume that $\left|w w^{\prime}\right|>|u|$, and denote $w w^{\prime}=u z_{3}$, where $z_{3} \in A_{\diamond}^{*}$. According to Theorem 2.2, the hole is in $w w^{\prime}$. We have $w^{\prime}=z_{1} z_{2} z_{3}, w=z_{1}^{\prime} z_{2}^{\prime} z_{3}^{\prime}, v=z_{1}^{\prime} z_{2}^{\prime} z_{3}^{\prime} z_{1}$ and $u=z_{1}^{\prime} z_{2}^{\prime} z_{3}^{\prime} z_{1} z_{2}$, where $z_{i}^{\prime} \uparrow z_{i}$ for all $i \in\{1,2,3\}$. Since $v \uparrow v^{\prime}$, we get that there exists $z_{4} \in A^{*}$ such that $z_{2} z_{3} z_{4}$ is a prefix of $v^{\prime}$ and $\left|z_{4}\right|=\left|z_{1}\right|$, and by looking at the prefixes of length $|w|$ of $u$ and $u^{\prime}$, we get that there exists $z_{5} \in A^{*}$, with $\left|z_{5}\right|=\left|z_{2}\right|$, such that $z_{1}^{\prime} z_{2}^{\prime} z_{3}^{\prime} \uparrow z_{3} z_{4} z_{5}$ (see Fig. 1). There are six cases to consider: Case 1 (respectively, Case 2, Case 3, Case 4, Case 5, Case 6) where the hole is in $z_{3}$ (respectively, $z_{2}$, $z_{1}, z_{3}^{\prime}, z_{2}^{\prime}, z_{1}^{\prime}$ ). We treat Cases 1,4 and 6 (Cases 2 and 3 are similar to Case 1 , and Case 5 to Case 4).

Case 1. The hole is in $z_{3}$.

We have $w^{\prime}=z_{1} z_{2} z_{3}, w=z_{1} z_{2} z_{3}^{\prime}, v=z_{1} z_{2} z_{3}^{\prime} z_{1}$ and $u=z_{1} z_{2} z_{3}^{\prime} z_{1} z_{2}$ where $z_{3} \subset z_{3}^{\prime}$. Since $z_{1} z_{2} z_{3}^{\prime}, z_{2} z_{3} z_{4}$ are prefixes of $v$ and $v^{\prime}$ respectively and $\left|z_{1} z_{2} z_{3}^{\prime}\right|=$ $\left|z_{2} z_{3} z_{4}\right|$, we get $z_{1} z_{2} z_{3}^{\prime} \uparrow z_{2} z_{3} z_{4}$, and $z_{1} z_{2} z_{3} \uparrow z_{2} z_{3} z_{4}$ by weakening. By Lemma 2.3, we get

$$
z_{1} z_{2} z_{3} z_{4} \text { is weakly }\left|z_{1}\right| \text {-periodic. }
$$

Now, since $w$ is without holes, the prefixes of length $|w|$ of $v$ and $v^{\prime}$, and respectively of $u$ and $u^{\prime}$ are compatible, and $z_{2} z_{3} z_{4} \subset w$ and $z_{3} z_{4} z_{5} \subset w$, we get $z_{2} z_{3} z_{4} \uparrow z_{3} z_{4} z_{5}$. Using Lemma 2.3 again, we get

$$
z_{2} z_{3} z_{4} z_{5} \text { is weakly }\left|z_{2}\right| \text {-periodic. }
$$

Finally, applying the weakening rule for the prefixes of length $|w|$ of $u$ and $u^{\prime}$, we get $z_{1} z_{2} z_{3} \uparrow z_{3} z_{4} z_{5}$. After using Lemma 2.3, we get

$$
z_{1} z_{2} z_{3} z_{4} z_{5} \text { is weakly }\left|z_{1} z_{2}\right| \text {-periodic. }
$$

From (2.1) and (2.3) we get that $z_{1} z_{2} z_{3} z_{4}$ is weakly $\left|z_{1}\right|$ - and weakly $\left|z_{1} z_{2}\right|$-periodic. Applying Lemma 2.1, we get that $z_{1} z_{2} z_{3} z_{4}$ is $\operatorname{gcd}\left(\left|z_{1}\right|,\left|z_{1} z_{2}\right|\right)$-periodic. Hence there 
exists a word $x \in A^{*}$ of length $\operatorname{gcd}\left(\left|z_{1}\right|,\left|z_{1} z_{2}\right|\right)$, such that $z_{1}=x^{m}$ and $z_{1} z_{2}=x^{m+n}$ for some integers $m, n>0$.

From (2.2) and (2.3) we get that $z_{2} z_{3} z_{4} z_{5}$ is weakly $\left|z_{2}\right|-$ and weakly $\left|z_{1} z_{2}\right|-$ periodic. Applying Lemma 2.1, we get that $z_{2} z_{3} z_{4} z_{5}$ is $\operatorname{gcd}\left(\left|z_{2}\right|,\left|z_{1} z_{2}\right|\right)$-periodic. Since $\operatorname{gcd}\left(\left|z_{1}\right|,\left|z_{1} z_{2}\right|\right)=\operatorname{gcd}\left(\left|z_{2}\right|,\left|z_{1} z_{2}\right|\right)$, we get that $z_{2} z_{3} z_{4} z_{5}$ is $|x|$-periodic. Because $\left|z_{1}\right| \geq|x|$ and $\left|z_{2}\right| \geq|x|$ we get that $z_{1} z_{2} z_{3} z_{4} z_{5}$ is $|x|$-periodic.

Because $z_{1}$ and $z_{5}$ share a prefix of length $\min \left(\left|x^{m}\right|,\left|x^{n}\right|\right)$ with $m, n>0, z_{5}$ is $|x|$-periodic and $\left|z_{5}\right|=\left|x^{n}\right|$, we get that $z_{5}=x^{n}=z_{2}$. Since $z_{3} z_{4} z_{5}$ is $|x|$-periodic, $\left|z_{5}\right| \geq|x|$ and $\left|z_{4}\right|=\left|x^{m}\right|$, we get that $z_{4}=x^{m}=z_{1}$.

Since $z_{1} z_{2} z_{3} z_{4}$ is $|x|$-periodic and $z_{1} z_{2}=x^{m+n}$, it results that $z_{3} \subset\left(x^{\prime} x^{\prime \prime}\right)^{p} x^{\prime}$ and $z_{4}=\left(x^{\prime \prime} x^{\prime}\right)^{m}$ where $x=x^{\prime} x^{\prime \prime}$ and $p \geq 0$ is an integer. But $z_{4}=z_{1}=x^{m}$. Hence, $x^{\prime} x^{\prime \prime}=x^{\prime \prime} x^{\prime}$ and there exists a word $y$, such that $x^{\prime}=y^{q}$ and $x^{\prime \prime}=y^{r}$ for some integers $q, r \geq 0$.

Since $v^{\prime} \uparrow v$, we have that $z_{2} z_{3} z_{1} z_{1} \uparrow z_{1} z_{2} z_{3}^{\prime} z_{1}$. By cancellation, we get $z_{2} z_{3} z_{1} \uparrow z_{1} z_{2} z_{3}^{\prime}$. Replacing $z_{1}$ by $x^{m}$ and $z_{2}$ by $x^{n}$, we get $x^{n} z_{3} x^{m} \uparrow x^{m} x^{n} z_{3}^{\prime}$, and consequently $z_{3} x^{m} \uparrow x^{m} z_{3}^{\prime}$ by cancellation. By Lemma 2.4, there exist words $y^{\prime}, y^{\prime \prime}$ such that $z_{3} \subset y^{\prime} y^{\prime \prime}, z_{3}^{\prime}=y^{\prime \prime} y^{\prime}$, and $x^{m}=\left(y^{\prime} y^{\prime \prime}\right)^{r} y^{\prime}$ for some integer $r \geq 0$. By Lemma 2.2, since $z_{3} \subset y^{\prime} y^{\prime \prime}$ and $z_{3} \subset z_{3}^{\prime}=y^{\prime \prime} y^{\prime}$, we get $y^{\prime} y^{\prime \prime}=y^{\prime \prime} y^{\prime}$. The latter implies that there exists a word $z$ such that $y^{\prime}$ and $y^{\prime \prime}$ are powers of $z$. We obtain $x^{m}=z^{m^{\prime}}$ for some integer $m^{\prime}$, and $x$ and $z$ are hence powers of a common word $z^{\prime}$. We conclude that $z_{1}, z_{2}, z_{3}, z_{3}^{\prime}, z_{4}$ and $z_{5}$ are contained in powers of $z^{\prime}$, implying that there is a later occurrence of a square compatible with $w^{2}$.

Case 4. The hole is in $z_{3}^{\prime}$.

Looking at the prefixes of length $|w|$ of $v$ and $v^{\prime}$, we have $z_{1} z_{2} z_{3}^{\prime} \uparrow z_{2} z_{3} z_{4}$. Applying weakening and Lemma 2.3, we get that $z_{1} z_{2} z_{3}^{\prime} z_{4}$ is weakly $\left|z_{1}\right|$-periodic. Also, by looking at the prefixes of length $|w|$ of $u$ and $u^{\prime}$ we get that $z_{1} z_{2} z_{3}^{\prime} \uparrow z_{3} z_{4} z_{5}$. We apply weakening and Lemma 2.3 again, and get that $z_{1} z_{2} z_{3}^{\prime} z_{4} z_{5}$ is weakly $\left|z_{1} z_{2}\right|$ periodic. Using Lemma 2.1, it follows that $z_{1} z_{2} z_{3}^{\prime} z_{4}$ is $\operatorname{gcd}\left(\left|z_{1}\right|,\left|z_{1} z_{2}\right|\right)$-periodic. Hence, there exists $x$ such that $z_{1}=x^{m}$ and $z_{1} z_{2}=x^{m+n}$, for some positive integers $m, n$, with $|x|=\operatorname{gcd}\left(\left|z_{1}\right|,\left|z_{1} z_{2}\right|\right)$. There exist $x^{\prime}, x^{\prime \prime}$ such that $x=x^{\prime} x^{\prime \prime}$, $z_{3}^{\prime} \subset\left(x^{\prime} x^{\prime \prime}\right)^{p} x^{\prime}$, for some integer $p \geq 0$, and $z_{4}=\left(x^{\prime \prime} x^{\prime}\right)^{m}$. Since the hole is in $z_{3}^{\prime}$, either there are integers $p_{1}, p_{2}$ and word $x_{1}^{\prime}$ having one hole such that $z_{3}^{\prime}=\left(x^{\prime} x^{\prime \prime}\right)^{p_{1}} x_{1}^{\prime}\left(x^{\prime \prime} x^{\prime}\right)^{p_{2}}$ with $x_{1}^{\prime} \subset x^{\prime}$ and $p_{1}+p_{2}=p$, or there are integers $p_{1}, p_{2}$ and word $x_{2}^{\prime}$ having one hole such that $z_{3}^{\prime}=\left(x^{\prime} x^{\prime \prime}\right)^{p_{1}} x^{\prime} x_{2}^{\prime}\left(x^{\prime} x^{\prime \prime}\right)^{p_{2}} x^{\prime}$ with $x_{2}^{\prime} \subset x^{\prime \prime}$ and $p_{1}+p_{2}+1=p$. Because $z_{3}^{\prime} \subset z_{3}$, it implies that either $z_{3}=\left(x^{\prime} x^{\prime \prime}\right)^{p_{1}} x_{1}\left(x^{\prime \prime} x^{\prime}\right)^{p_{2}}$ with $x_{1}^{\prime} \subset x_{1}$, or $z_{3}=\left(x^{\prime} x^{\prime \prime}\right)^{p_{1}} x^{\prime} x_{2}\left(x^{\prime} x^{\prime \prime}\right)^{p_{2}} x^{\prime}$ with $x_{2}^{\prime} \subset x_{2}$.

But also, $z_{1} z_{2} z_{3}^{\prime} \uparrow z_{2} z_{3} z_{4}$. Hence, we get that $x^{m} z_{3}^{\prime} \uparrow z_{3} z_{4}$. This is equivalent to one of the following cases:

$$
x^{m}\left(x^{\prime} x^{\prime \prime}\right)^{p_{1}} x_{1}^{\prime}\left(x^{\prime \prime} x^{\prime}\right)^{p_{2}} \uparrow\left(x^{\prime} x^{\prime \prime}\right)^{p_{1}} x_{1}\left(x^{\prime \prime} x^{\prime}\right)^{p_{2}}\left(x^{\prime \prime} x^{\prime}\right)^{m}
$$

when we get $x_{1}=x^{\prime}$, or

$$
x^{m}\left(x^{\prime} x^{\prime \prime}\right)^{p_{1}} x^{\prime} x_{2}^{\prime}\left(x^{\prime} x^{\prime \prime}\right)^{p_{2}} x^{\prime} \uparrow\left(x^{\prime} x^{\prime \prime}\right)^{p_{1}} x^{\prime} x_{2}\left(x^{\prime} x^{\prime \prime}\right)^{p_{2}} x^{\prime}\left(x^{\prime \prime} x^{\prime}\right)^{m}
$$


when we get $x_{2}=x^{\prime \prime}$. In either case, $z_{3}=\left(x^{\prime} x^{\prime \prime}\right)^{p} x^{\prime}$.

Since $z_{1} z_{2} z_{3}^{\prime} \uparrow z_{3} z_{4} z_{5}$, there is the possibility that $z_{5}=\left(x^{\prime \prime} x^{\prime}\right)^{n}$ if $n \leq p_{2}$. We leave this case to the reader and assume that $n>p_{2}$. We get that $z_{5}=$ $\left(x^{\prime \prime} x^{\prime}\right)^{n_{1}} x^{\prime \prime} x_{1}\left(x^{\prime \prime} x^{\prime}\right)^{n_{2}}$ with $x_{1}^{\prime} \subset x_{1}$, or $z_{5}=\left(x^{\prime \prime} x^{\prime}\right)^{n_{1}} x_{2} x^{\prime}\left(x^{\prime \prime} x^{\prime}\right)^{n_{2}}$ with $x_{2}^{\prime} \subset x_{2}$ (in either case $n_{1}+n_{2}+1=n$ ). Since $v \uparrow v^{\prime}$, it follows that $z_{5}$ and $z_{1}$ share a prefix of length $|x|$, and so $z_{5}$ has $x^{\prime} x^{\prime \prime}$ as a prefix. There are three cases to consider: (4.1) $x^{\prime} x^{\prime \prime}=x^{\prime \prime} x^{\prime}$; (4.2) $x^{\prime} x^{\prime \prime}=x^{\prime \prime} x_{1}$; and (4.3) $x^{\prime} x^{\prime \prime}=x_{2} x^{\prime}$. For (4.1), there exists a word $y$ such that $x^{\prime}$ and $x^{\prime \prime}$ are powers of $y$. It follows that $z_{1}, z_{2}, z_{3}, z_{3}^{\prime}$ and $z_{4}$ are contained in powers of $y$, implying that there is a later occurrence of a partial word that is compatible with the square $\left(w^{\prime}\right)^{2}$. For (4.2) and (4.3), $n_{1}=0$ and we can denote $z_{5}$ as $x^{\prime} x^{\prime \prime}\left(x^{\prime \prime} x^{\prime}\right)^{n-1}$. Furthermore, since $z_{1} z_{2} z_{3}^{\prime} \uparrow z_{3} z_{4} z_{5}$ we get that either $x^{m+n+p_{1}} x_{1}^{\prime}\left(x^{\prime \prime} x^{\prime}\right)^{p_{2}} \uparrow x^{m+p} x^{\prime} x^{\prime} x^{\prime \prime}\left(x^{\prime \prime} x^{\prime}\right)^{n-1}$ or $x^{m+n+p_{1}} x^{\prime} x_{2}^{\prime}\left(x^{\prime} x^{\prime \prime}\right)^{p_{2}} x^{\prime} \uparrow x^{m+p} x^{\prime} x^{\prime} x^{\prime \prime}\left(x^{\prime \prime} x^{\prime}\right)^{n-1}$. We prove the first case (the other is similar).

If $p>n+p_{1}$, then $p_{2}>n$ a contradiction. If $p=n+p_{1}$, then $x_{1}^{\prime} x^{\prime \prime} x^{\prime} \uparrow x^{\prime} x^{\prime} x^{\prime \prime}$ and $x^{\prime} x^{\prime \prime}=x^{\prime \prime} x^{\prime}$, the same contradiction as before follows. If $p<n+p_{1}$, we get $x^{n-p_{2}} x_{1}^{\prime} \uparrow x^{\prime} x^{\prime} x^{\prime \prime}\left(x^{\prime \prime} x^{\prime}\right)^{n-1-p_{2}}$. If $p_{2}<n-1$, then again $x^{\prime} x^{\prime \prime}=x^{\prime \prime} x^{\prime}$. If $p_{2}=n-1$, then $x^{\prime} x^{\prime \prime} \uparrow x^{\prime \prime} x_{1}^{\prime}$. By Lemma 2.4, there exist words $y^{\prime}, y^{\prime \prime}$ such that $x^{\prime}=y^{\prime} y^{\prime \prime}, x_{1}^{\prime} \subset y^{\prime \prime} y^{\prime}$, and $x^{\prime \prime}=\left(y^{\prime} y^{\prime \prime}\right)^{r} y^{\prime}$ for some integer $r \geq 0$. By Lemma 2.2, since $x_{1}^{\prime} \subset y^{\prime \prime} y^{\prime}$ and $x_{1}^{\prime} \subset x^{\prime}=y^{\prime} y^{\prime \prime}$, we get $y^{\prime} y^{\prime \prime}=y^{\prime \prime} y^{\prime}$. The latter implies that there exists a word $z$ such that $y^{\prime}$ and $y^{\prime \prime}$ are powers of $z$. We obtain $x^{\prime}$ and $x^{\prime \prime}$ are powers of $z$. We conclude that $z_{1}, z_{2}, z_{3}, z_{3}^{\prime}, z_{4}$ and $z_{5}$ are contained in powers of $z$, implying that there is a later occurrence of a square compatible with $\left(w^{\prime}\right)^{2}$.

Case 6. The hole is in $z_{1}^{\prime}$.

Since $v \uparrow v^{\prime}, z_{1}^{\prime} z_{2} z_{3}$ and $z_{2} z_{3} z_{4}$ are prefixes of $v$ and $v^{\prime}$ respectively, and $\left|z_{1}^{\prime} z_{2} z_{3}\right|=\left|z_{2} z_{3} z_{4}\right|$, we have that $z_{1}^{\prime} z_{2} z_{3} \uparrow z_{2} z_{3} z_{4}$. Applying Lemma 2.4, we get that $z_{1}^{\prime} z_{2} z_{3} z_{4}$ is $\left|z_{1}\right|$-periodic. Since $u \uparrow u^{\prime}$, we get that $z_{1}^{\prime} z_{2} z_{3} \uparrow z_{3} z_{4} z_{5}$, and $z_{1}^{\prime} z_{2} z_{3} z_{4} z_{5}$ is $\left|z_{1} z_{2}\right|$-periodic. Hence, $z_{1}^{\prime} z_{2} z_{3} z_{4}$ is $|x|=\operatorname{gcd}\left(\left|z_{1}\right|,\left|z_{1} z_{2}\right|\right)$-periodic, where $z_{1}^{\prime} \subset x^{m}$ and $z_{2}=x^{n}$, for some word $x$ and integers $m, n>0$. This implies that there exist $x^{\prime}, x^{\prime \prime} \in A^{*}$, such that $x=x^{\prime} x^{\prime \prime}$, and $z_{3}=\left(x^{\prime} x^{\prime \prime}\right)^{p} x^{\prime}$, for some integer $p \geq 0, z_{4}=\left(x^{\prime \prime} x^{\prime}\right)^{m}$ and $z_{5}=\left(x^{\prime \prime} x^{\prime}\right)^{n}$ (because $z_{2} z_{3} z_{4} z_{5}$ is $\left|z_{1} z_{2}\right|$-periodic and $\left.\left|z_{2} z_{3} z_{4}\right|>\left|z_{1} z_{2}\right|\right)$.

Since $v \uparrow v^{\prime}$, if $\left|z_{2}\right| \geq\left|z_{1}\right|$, then $z_{5}$ and $z_{1}$ share a common prefix of length $\left|x^{m}\right|$. It follows that $z_{1}=\left(x^{\prime \prime} x^{\prime}\right)^{m}$. But, since $z_{1}^{\prime} \subset z_{1}$, it results that $z_{1}^{\prime} \subset\left(x^{\prime \prime} x^{\prime}\right)^{m}$, and recall that $z_{1}^{\prime} \subset\left(x^{\prime} x^{\prime \prime}\right)^{m}$. If $m>1$, then we get $x^{\prime} x^{\prime \prime}=x^{\prime \prime} x^{\prime}$ and so there exists $y$, such that $x^{\prime}=y^{q}$ and $x^{\prime \prime}=y^{r}$ for some nonnegative integers $q, r$, giving us a contradiction with the assumption that there is no later occurrence of a factor compatible with $\left(w^{\prime}\right)^{2}$. If $m=1$, then we also get $x^{\prime} x^{\prime \prime}=x^{\prime \prime} x^{\prime}$ by Lemma 2.2. Hence, we may assume that $\left|z_{2}\right|<\left|z_{1}\right|$ and $z_{1}$ has as a prefix $\left(x^{\prime \prime} x^{\prime}\right)^{n}$. Let $z_{6} \in A^{*}$, where $\left|z_{6}\right|=\left|z_{2}\right|$, such that $z_{1}^{\prime} z_{2} z_{3} z_{1} \uparrow z_{3} z_{4} z_{1} z_{6}$ (the prefixes of length $|v|$ of $u$ and $u^{\prime}$ are compatible). By using simplification we get that $z_{2} x^{\prime} z_{1} \uparrow x^{\prime} z_{1} z_{6}$ and $z_{2} x^{\prime} z_{1} z_{6}$ is $\left|z_{2}\right|$-periodic by Lemma 2.4. Since $\left|z_{1}\right|=\left|x^{m}\right|>\left|z_{2}\right|$, it follows that $z_{1}=\left(x^{\prime \prime} x^{\prime}\right)^{m}$. Since $z_{1}^{\prime} \subset\left(x^{\prime} x^{\prime \prime}\right)^{m}$, we get a contradiction as before. 
Since Cases 1-6 lead to contradiction we conclude that $z_{3}=\varepsilon$.

Let us now assume that the hole is at a position $i$ in a word of length $n$. The upper bound for the maximum number of factors, compatible with distinct squares, would be achieved if all these factors, starting before the hole, would contain the hole (then more than two squares can start at the same position). Note that in the case when a square containing a hole has its last occurrence at a certain position, no other word that is a square can have its last occurrence starting at the same position (otherwise a later occurrence of the same word, or a word compatible with it, would appear later in the word). Let us look at the start position $j$ of a square containing the hole and denote it as $j$ (obviously, there are at most $i$ such squares). Let us denote the length of such square by $n_{j}$.

Hence, if at position $j$ we have a square of length $n_{j}$, then according to Theorem 2.3, up to position $2 n_{j}+j$ we will have counted at most three distinct squares. Using an induction we notice that up to position $2^{m} n_{j}+j$ we will have counted at most $2 m+1$ distinct squares. Since the length of the word is $n$, we have that the maximum value for $m$, for squares starting at position $j$, is bounded by $\log \left(\frac{n-j}{n_{j}}\right)$.

Note that the maximum is achieved for the case when $n_{j}$ is minimum. Hence we can replace in our formula $n_{j}$ by $i-j$, which is the smallest length a square starting at position $j$ and containing the hole may have.

Theorem 2.4. The number of distinct squares compatible with factors in any partial word with one hole of length $n$ is at most $\frac{7 n}{2}$.

Proof. Using the previous remarks it is easy to see that the number of squares at position $j$ and containing the hole is $2 \log \left(\frac{n-j}{i-j}\right)+1$. Hence, we get that the total number of distinct squares that we can obtain is

$$
\sum_{j=0}^{i-1}\left(2 \log \left(\frac{n-j}{i-j}\right)+1\right)=i+\frac{2}{\ln 2} \sum_{j=0}^{i-1} \ln \left(\frac{n-j}{i-j}\right) .
$$

The sum from the previous formula is equal to $\sum_{x=n-i+1}^{n} \ln (x)-\sum_{y=1}^{i} \ln (y)$ and implicitly, less or equal than $\int_{n-i+1}^{n+1} \ln (x) \mathrm{d} x-\int_{1}^{i} \ln (y) \mathrm{d} y$. After integrating we get

$$
(n+1) \ln (n+1)-(n-i+1) \ln (n-i+1)-i \ln (i) .
$$

Since the maximum is obtained for $i=\frac{n+1}{2}$, the function is hence less than $(n+$ 1) $\ln 2$. Using Theorem 2.1 for the rest of the word, we get that the number of distinct squares, compatible with factors of the word, is bounded by

$$
2 n-\frac{n+1}{2}+\frac{2}{\ln 2}(n+1) \ln 2=\frac{7 n}{2}+\frac{3}{2} .
$$

Since the last position in the word contains no squares, we get that the maximum number of factors compatible with distinct squares is smaller than $\frac{7 n}{2}$.

This bound can be slightly improved by using Ilie's $2 n-\Theta(\log n)$. 


\section{Conclusion}

In order to improve the bound stated in Theorem 2.4, we need to somehow limit to less than 3.5 the average number of squares that have their last occurrence starting at the positions of the partial word. This requirement draws attention to positions $i$ where three or more squares have their last occurrences. Is it true that at positions "neighbouring" to $i$, no squares can have their last occurrences? In fact, if at position $i$ we have at least three factors compatible with squares, this does not imply that at position $i+1$ we will have less. Indeed, consider the example

\section{$a b \diamond a b c a b b e a b c a b d a b c a b b e a b c a b b a b c a b b e a b c a b d a b c a b b e a b c a b$}

where at position 0 we have factors compatible with the squares

$$
\left.(a b c)^{2},(a b d a b c a b b e a b c)^{2} \text { and (abbabcabbeabcabdabcabbeabc }\right)^{2}
$$

at position 1 factors compatible with the squares

$$
\left.(b c a)^{2},(\text { beabcab })^{2},(\text { bdabcabbeabca })^{2} \text { and (bbabcabbeabcabdabcabbeabca }\right)^{2}
$$

and at position 2 factors compatible with

$$
\left.a^{2},(c a b)^{2},(\text { dabcabbeabcab })^{2} \text { and (babcabbeabcabdabcabbeabcab }\right)^{2} .
$$

In [8], Ilie gave a relation between the lengths of squares at positions neighbouring a position where two squares have their last occurrences. More precisely, he showed that if $v^{2}<u^{2}$ are two squares at position $i$ and $w^{2}$ is a square at position $i+1$, then either $|w| \in\{|v|,|u|\}$ or $|w| \geq 2|v|$ (see Lem. 2 of [8]). Referring to the above example, we observe that such is not the case with partial words with one hole.

\section{REFERENCES}

[1] J. Berstel and L. Boasson, Partial words and a theorem of Fine and Wilf. Theoret. Comput. Sci. 218 (1999) 135-141.

[2] F. Blanchet-Sadri, Algorithmic Combinatorics on Partial Words. Chapman \& Hall/CRC Press, Boca Raton, FL (2008).

[3] F. Blanchet-Sadri and D.K. Luhmann, Conjugacy on partial words. Theoret. Comput. Sci. 289 (2002) 297-312.

[4] F. Blanchet-Sadri, R. Mercaş and G. Scott, Counting distinct squares in partial words, edited by E. Csuhaj-Varju, Z. Esik, AFL 2008, 12th International Conference on Automata and Formal Languages, Balatonfüred, Hungary (2008) 122-133, www.uncg.edu/cmp/research/ freeness

[5] F. Blanchet-Sadri, D.D. Blair and R.V. Lewis, Equations on partial words. RAIRO-Theor. Inf. Appl. 43 (2009) 23-39, www.uncg.edu/cmp/research/equations

[6] A.S. Fraenkel and J. Simpson, How many squares can a string contain? J. Combin. Theory Ser. A 82 (1998) 112-120.

[7] L. Ilie, A simple proof that a word of length $n$ has at most $2 n$ distinct squares. J. Combin. Theory Ser. A 112 (2005) 163-164.

[8] L. Ilie, A note on the number of squares in a word. Theoret. Comput. Sci. 380 (2007) 373-376.

Communicated by C. Choffrut.

Received July 23, 2008. Accepted August 18, 2009. 